\title{
Statistics of point vortex turbulence in non-neutral flows and in flows with translational and rotational symmetries
}

\author{
J. G. Esler
}

Received: date / Accepted: date

\begin{abstract}
A theory [Esler and Ashbee, J. Fluid Mech., 779, 275, 2015] describing the statistics of $N$ freely-evolving point vortices in a bounded twodimensional domain is extended. First, the case of a non-neutral vortex gas is addressed, and it is shown that the density of states function can be identified with the probability density function of an infinite sum of independent non-central chi-squared random variables, the details of which depend only on the shape of the domain. Equations for the equilibrium energy spectrum and other statistical quantities follow, the validity of which are verified against direct numerical simulations of the equations of motion. Second, domains with additional conserved quantities associated with a symmetry (e.g. circle, periodic channel) are investigated, and it is shown that the treatment of the non-neutral case can be modified to account for the additional constraint.
\end{abstract}

Keywords Point vortices · Energy spectrum

\section{Introduction}

Interest in the classical statistical mechanics of point vortices, as developed by Onsager [18], Joyce and Montgomery [15], Pointin and Lundgren [19] and others, has been recently revived by experimental developments in quantum fluids, e.g. [17]. In quantum fluids (e.g. Bose-Einstein condensates), vortices have quantised circulations, and in experiments where the 'healing length' of these vortices is much less than the mean free path between them, the point vortex model can be expected accurately capture their essential dynamics [20].

Supported by the Leverhulme Trust RF-2016-158 and Kavli Institute NSF PHY11-25915

J.G. Esler

Department of Mathematics

University College London

Gower St., London

E-mail: j.g.esler@ucl.ac.uk 
Point vortex statistical mechanics has been used to interpret phenomena such as energy spectrum evolution [2] and condensate formation [22,21] observed in numerical simulations. This research, as well as the continued relevance of vortex models of classical 2D turbulence [10], motivate the current study.

In a recent work, Esler and Ashbee [12] (EA15 hereafter) developed a quantitative approach to the statistical mechanics of $N$ point vortices of arbitrary ${ }^{1}$ circulations in a general simply-connected bounded domain $\mathcal{D} \subset \mathbb{R}^{2}$, in the limit $N \rightarrow \infty$. The case of the doubly-periodic domain has also been addressed in [11]. EA15 was concerned with the neutral case, for which the vortex circulations $\left(\Gamma_{1}, \ldots, \Gamma_{N}\right)$ are constrained to satisfy

$$
\sum_{i=1}^{N} \Gamma_{i}=0 .
$$

The main results of EA15 can be summarised:

1. A systematic method for calculating the density of states function $W(\varepsilon)$, in the limit $N \rightarrow \infty$, for an arbitrary domain and general distribution of vortices was found. The density of states is a measure of the number of vortex arrangements (microstates) consistent with a fixed (and appropriately scaled) Hamiltonian energy level $\varepsilon$. The resulting formula for $W(\varepsilon)$ is independent of the details of the vortex circulations, a result consistent with the statistical sampling study of Campbell and O'Neil [4].

2. It was shown that the energy spectrum of the point vortex flow, which depends only on the energy level $\varepsilon$, is wholly determined by $W(\varepsilon)$. Specifically,

$$
\left\langle E_{j}\right\rangle=\frac{1}{2 W(\varepsilon)} \int_{-\infty}^{\varepsilon} W(\bar{\varepsilon}) \mathrm{e}^{\beta_{j}(\varepsilon-\bar{\varepsilon})} \mathrm{d} \bar{\varepsilon} .
$$

Here $E_{j}(t)$ is the energy contained in the $j$ th 'hydrodynamic' eigenmode of the domain $\mathcal{D}$ (see Appendix A for definitions), and $\left\langle E_{j}\right\rangle$ is its expected long-time average under the dynamics. To obtain (2), an ergodic hypothesis is assumed to hold, under which the dynamical time-average is equivalent to the microcanonical ensemble average. The negative constant $\beta_{j}$ is the (scaled) eigenvalue of the $j$ th eigenmode, which can be related to the eigenmode wavenumber $k_{j}$ by $k_{j}=\left(-\beta_{j} / A\right)^{1 / 2}$ where $A=|\mathcal{D}|$ is the domain area.

3. The validity of the new statistical theory was demonstrated by comparison with numerical simulations of the point vortex equations with $N=100$. Consistent with the use of the central limit theorem (CLT) in its derivation, the theory is accurate provided that $\left|\varepsilon / \sigma_{\varepsilon}\right| \lesssim N^{1 / 2}$, where $\sigma_{\varepsilon}$ is the standard deviation of the density of states $W(\varepsilon)$.

4. The connection with the mean field theory of Joyce and Montgomery [15], was made explicit. The EA15 theory (see also [19]) is recovered under 'thermodynamic scaling', which for vortices of equal circulation means that $\varepsilon$

1 Under some mild restrictions, see EA15. 
is the Hamiltonian energy per vortex. The mean-field theory is obtained under 'hydrodynamic scaling' in which the relevant energy $E$ is proportional to the Hamiltonian energy divided by $N^{2}$. (Equivalently, in the notation of the current paper, the mean-field theory is formally valid for $0<\varepsilon / \sigma_{\varepsilon} \sim N$.) That the two theories are consistent can be demonstrated by asymptotic matching: the $\varepsilon \rightarrow \infty$ limit of EA15 reveals the emergence of a condensate (or mean-field) into which all excess energy is concentrated. The structure of this condensate turns out to be exactly identical to the solution of the mean-field equation (sinh-Poisson) equation in the low energy limit $E \rightarrow 0$ of the mean-field theory.

The aim of the present work is to extend the results of EA15 to:

(A) Non-neutral distributions of vortices in which the constraint (1) no longer holds. In this case there is a non-zero circulation in $\mathcal{D}$ which requires significant modifications to the analysis of EA15.

(B) Domains with a geometric symmetry associated with an additional conserved quantity. Important examples include the circle and the annulus, in which angular momentum is conserved, and the periodic channel with sidewalls, which conserves linear $(x)$-momentum. Other examples are possible if the extension to general two-dimensional surfaces is considered [8].

It will be shown below that it is natural to treat (A) and (B) in a similar fashion.

The plan of the work is as follows. In section 2 the extended theory is set out for the non-neutral case, and theoretical predictions are compared with both direct numerical simulations (DNS) of the point vortex dynamics with $N=100$ vortices, and statistical sampling of the microcanonical ensemble. In section 3 the theory is extended to domains with additional conserved quantities, and the specific example of the unit circle is chosen for detailed examination and comparison with DNS. In section 4 conclusions are drawn.

\section{General domain: The non-neutral case}

\subsection{Set-up and definitions}

Consider the general situation of $N$ point vortices, with circulations $\left(\Gamma_{1}, \ldots, \Gamma_{N}\right)$ and positions $\left(\boldsymbol{x}_{1}(t), \ldots, \boldsymbol{x}_{N}(t)\right)$ evolving in a bounded and simply-connected domain $\mathcal{D} \subset \mathbb{R}^{2}$ of area $A$. (Results are straightforward to adapt to the nonconformal domains mentioned above, namely the annulus and periodic channel.) Denoting the domain average over $\mathcal{D}$ of any function $f(\boldsymbol{x})$ by

$$
[f]=\frac{1}{A} \int_{\mathcal{D}} f(\boldsymbol{x}) \mathrm{d} \boldsymbol{x},
$$

it is evident that the vorticity distribution

$$
\omega(\boldsymbol{x}, t)=\sum_{i=1}^{N} \Gamma_{i} \delta\left(\boldsymbol{x}-\boldsymbol{x}_{i}(t)\right),
$$


has a constant domain average

$$
[\omega]=\frac{1}{A} \sum_{i=1}^{N} \Gamma_{i}
$$

Introducing the r.m.s. circulation

$$
\Gamma_{0}=\left(\frac{1}{N} \sum_{i=1}^{N} \Gamma_{i}^{2}\right)^{1 / 2}
$$

it turns out that all the behavior of interest can be captured by concentrating on the regime with $[\omega] \sim \Gamma_{0} N^{1 / 2}$. In this 'weakly non-neutral' regime the energy in the mean flow, associated with the domain-averaged vorticity $[\omega]$, is comparable to the energy in the fluctuations. In strongly non-neutral flows (i.e. $\left.[\omega] \sim \Gamma_{0} N\right)$, by contrast, the energy is almost entirely in the mean flow, as can be seen by taking the limit $\Omega_{0} \rightarrow \infty$ within the weakly non-neutral theory.

Following EA15, the next step is to expand the vorticity field in a Fourier series based on the hydrodynamic basis [14] $\left\{\Phi_{j}(\boldsymbol{x}), j \geq 0\right\}$ of $\mathcal{D}$, which is introduced in detail in Appendix A. A key property of the hydrodynamic basis is that its leading eigenfunction is $\Phi_{0}(\boldsymbol{x})=1$ and that all subsequent eigenfunctions are orthogonal to $\Phi_{0}$. As a consequence, the hydrodynamic basis is well-suited to represent functions with a conserved domain average, such as the vorticity field $\omega$ under consideration here. It is helpful to introduce the vorticity scale $\omega_{0}=N^{1 / 2} \Gamma_{0} / A$ and to use completeness to expand

$$
\frac{\omega(\boldsymbol{x}, t)}{\omega_{0}}=\Omega_{0}+\sum_{j=1}^{\infty} \Omega_{j}(t) \Phi_{j}(\boldsymbol{x}) .
$$

It follows, from orthogonality, that $\Omega_{j}=\left[\omega \Phi_{j}\right] / \omega_{0}$. In particular, $\Omega_{0}=[\omega] / \omega_{0}$ is an order unity constant for a weakly non-neutral distribution of vortex circulations, with the strongly non-neutral case accessible in the limit $\Omega_{0} \rightarrow$ $\pm \infty$.

In the procedure of EA15, the expansion for $\omega$ is inserted into the equation for the point vortex Hamiltonian [16],

$$
H\left(\boldsymbol{x}_{1}, \ldots, \boldsymbol{x}_{N}\right)=-\frac{1}{2} \sum_{i=1}^{N} \sum_{j=1, j \neq i}^{N} \Gamma_{i} \Gamma_{j} G\left(\boldsymbol{x}_{i}, \boldsymbol{x}_{j}\right)-\frac{1}{2} \sum_{i=1}^{N} \Gamma_{i}^{2} r\left(\boldsymbol{x}_{i}\right) .
$$

Here $G\left(\boldsymbol{x}, \boldsymbol{x}^{\prime}\right)$ is the (Dirichlet) Green's function for $\mathcal{D}$ and $r(\boldsymbol{x})$ is the Robin function

$$
r(\boldsymbol{x})=\lim _{\boldsymbol{x}^{\prime} \rightarrow \boldsymbol{x}}\left(G\left(\boldsymbol{x}, \boldsymbol{x}^{\prime}\right)-\frac{1}{2 \pi} \log \left|\boldsymbol{x}-\boldsymbol{x}^{\prime}\right|\right) .
$$

To facilitate inserting the expansion for $\omega,(8)$ is first rewritten as

$$
H=-\frac{1}{2} \int_{\mathcal{D}^{2}}\left(\omega(\boldsymbol{x}) \omega\left(\boldsymbol{x}^{\prime}\right)-R\left(\boldsymbol{x}, \boldsymbol{x}^{\prime}\right)\right) G\left(\boldsymbol{x}, \boldsymbol{x}^{\prime}\right) \mathrm{d} \boldsymbol{x} \mathrm{d} \boldsymbol{x}^{\prime}-\frac{1}{2} \int_{\mathcal{D}} S(\boldsymbol{x}) r(\boldsymbol{x}) \mathrm{d} \boldsymbol{x} .
$$


where $R\left(\boldsymbol{x}, \boldsymbol{x}^{\prime}\right)$ and $S(\boldsymbol{x})$ are the distributions

$$
R\left(\boldsymbol{x}, \boldsymbol{x}^{\prime}\right)=\sum_{i=1}^{N} \Gamma_{i}^{2} \delta\left(\boldsymbol{x}-\boldsymbol{x}_{i}\right) \delta\left(\boldsymbol{x}^{\prime}-\boldsymbol{x}_{i}\right), \quad S(\boldsymbol{x})=\sum_{i=1}^{N} \Gamma_{i}^{2} \delta\left(\boldsymbol{x}-\boldsymbol{x}_{i}\right) .
$$

To proceed, the Dirichlet Green's function $G\left(\boldsymbol{x}, \boldsymbol{x}^{\prime}\right)$ is expanded in the hydrodynamic basis, as described ${ }^{2}$ in detail in Appendix A. Following EA15, where further detail is provided, the expansions (7) and (44) are inserted into (10), along with analogous expansions for $R$ and $S$. Eigenfunction orthogonality is then used to evaluate the resulting integrals. Finally, the limit $N \rightarrow \infty$ is taken, and terms of relative magnitude $O(1 / N)$ are neglected. The result is

$$
\bar{H} \equiv \frac{H}{N \Gamma_{0}^{2}}=\varepsilon_{0}\left(\Omega_{0}\right)+\sum_{j=1}^{\infty} \frac{\left(\Omega_{j}+\mu_{j} \Omega_{0}\right)^{2}-\mu_{j}^{2} \Omega_{0}^{2}-1}{-2 \beta_{j}} .
$$

Notice that in (12) the classical point vortex Hamiltonian $H$ has been rescaled by a factor $N \Gamma_{0}^{2}$. The scaled Hamiltonian $\bar{H}$ is convenient to work with, as it remains invariant under a rescaling of the vortex circulations, and is also independent of $N$ in the limit $N \rightarrow \infty$. The function $\varepsilon_{0}\left(\Omega_{0}\right)$ and sequences of constants $\left\{\beta_{j}\right\}$ and $\left\{\mu_{j}\right\}$ appearing in (12) are

$$
\varepsilon_{0}\left(\Omega_{0}\right)=\frac{G_{00}\left(1-\Omega_{0}^{2}\right)-r_{0}}{2}, \quad \beta_{j}=\frac{1}{G_{j j}}, \quad \mu_{j}=\frac{G_{0 j}}{G_{j j}},
$$

where

$$
G_{j k}=\left[\left[\Phi_{j}(\boldsymbol{x}) G\left(\boldsymbol{x}, \boldsymbol{x}^{\prime}\right) \Phi_{k}\left(\boldsymbol{x}^{\prime}\right)\right]\right]^{\prime} \text { and } r_{j}=\left[\Phi_{j}(\boldsymbol{x}) r(\boldsymbol{x})\right],
$$

where $[[\cdot]]^{\prime}$ denotes the double domain average over both the $\boldsymbol{x}$ and $\boldsymbol{x}^{\prime}$ coordinates. Conformal mapping techniques can be used to show that the $\left\{G_{j k}\right\}$ depend only on the shape of $\mathcal{D}$ (i.e. they are independent of its area $A$ ). For the case of domains which are not simply-connected, such as the annulus, the same result can be recovered by using the Schottky-Klein prime function [7] to obtain the Green's function. It follows that the only area-dependence in (12) is through the constant $r_{0}$, which under a rescaling of the domain area $A \rightarrow \alpha^{2} A$ transforms according to $r_{0} \rightarrow r_{0}-(1 / 2 \pi) \log \alpha$. It follows that the domain area, as opposed to the domain shape, does not affect the vortex statistics except for a (trivial) change in the value of the constant $\varepsilon_{0}$. For a domain $\mathcal{D}$ with a known or calculable conformal map to the unit circle, the constants $\left\{\beta_{j}\right\},\left\{\mu_{j}\right\}$ and $r_{0}$ can obtained to high accuracy, up to some finite $j=J$, using spectral methods [24]. For the rectangle and circle (see below) analytical results are available $[6,23]$.

The terms in the summation (12) have a useful interpretation in terms of the energetics of the point vortex flow. Defining

$$
E_{j}=-\frac{\left(\Omega_{j}+\mu_{j} \Omega_{0}\right)^{2}}{2 \beta_{j}} \quad \text { and } \quad E_{0 j}=-\frac{1+\mu_{j}^{2} \Omega_{0}^{2}}{2 \beta_{j}},
$$

\footnotetext{
2 In fact, there is an error in the corresponding expansion in EA15, their eqn. 3.9a should be equation (44) here. The error is inconsequential for the results in EA15, however the use of the correct form (44) is essential here.
} 

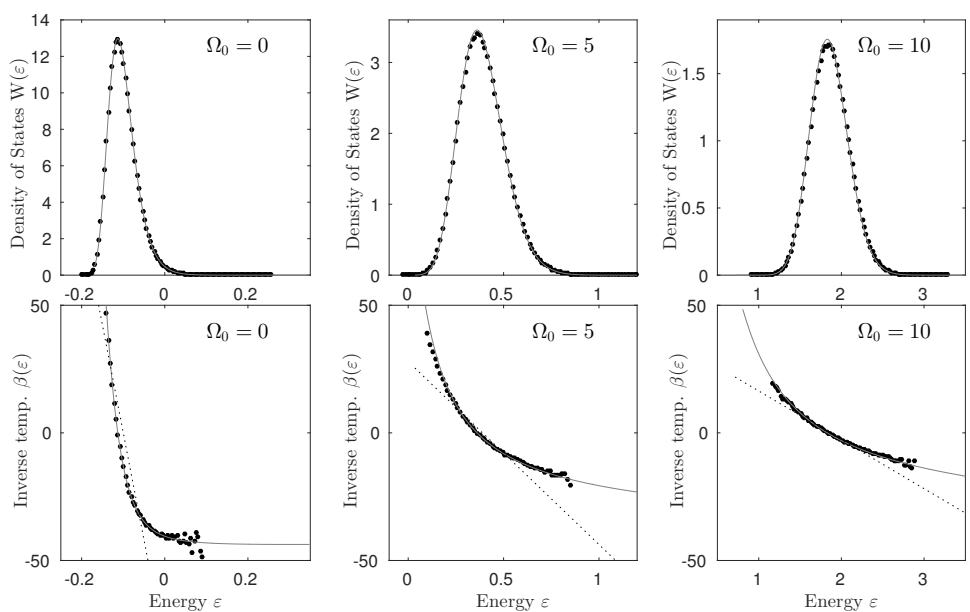

Fig. 1 Upper panels: Density of states $W\left(\varepsilon, \Omega_{0}\right)$ for domain $\mathrm{A}$, for $\Omega_{0}=0,5,10$. Curves are theoretical predictions calculated from (18), and points are calculated from a histogram of $\bar{H}$ under the uniform ensemble $(N=100$ vortices randomly placed in $\mathcal{D}$ under uniform measure). The sample size for $\bar{H}$ is $10^{6}$. Lower panels: Inverse temperature $\beta\left(\varepsilon, \Omega_{0}\right)$ for domain A, for $\Omega=0,5,10$. The dotted curves show the Gaussian approximation (20).

$E_{j}$ is the (scaled) fluid dynamical energy in the $j$ th mode (see Appendix A.1 for discussion). Consistent with the fact that the fluid dynamical energy of a single point vortex is singular, the sum $\sum_{j} E_{j}$ is divergent. However the energy spectrum, as defined by the $\left\{E_{j}\right\}$, remains well-defined [9]. The constants $E_{0 j}$ are easily shown to be equal to the expected energy in the $j$ th mode, had the vortices been distributed randomly in $\mathcal{D}$ under uniform measure. The difference $E_{j}^{\prime}=E_{j}-E_{0 j}$ is the anomalous energy in the $j$ th mode, and from (12) it is evident that the Hamiltonian $\bar{H}$ is equal to the sum of anomalous energies over all modes (up to a constant).

\subsection{Density of states function and caloric curves}

The density of states function is a measure of the number of microstates (vortex arrangements) associated with the hypersurface in phase-space defined by $\bar{H}=$ $\varepsilon$. Next, a method for calculating it will be presented, which will extend the results of EA15 to non-neutral vortex distributions. Following normalisation, the density of states can be identified with the pdf of $\bar{H}$ under the uniform ensemble, in which all vortices are distributed randomly in $\mathcal{D}$ under uniform measure. From (12), it follows that for a non-neutral vortex gas, the density of states $W\left(\varepsilon, \Omega_{0}\right)$ will depend only on $\varepsilon$ and $\Omega_{0}$, and not on any other details of the vortex circulations. The normalisation for $W\left(\varepsilon, \Omega_{0}\right)$ to be a pdf is

$$
\int_{-\infty}^{\infty} W\left(\varepsilon, \Omega_{0}\right) \mathrm{d} \varepsilon=1
$$


A

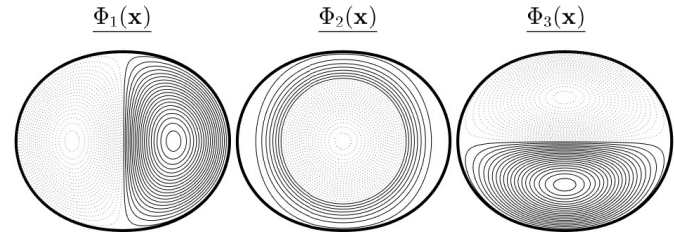

B
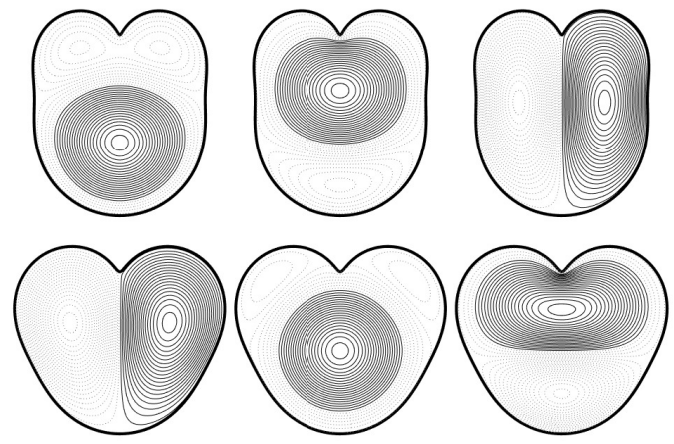

Fig. 2 Hydrodynamic modes $\Phi_{1}, \Phi_{2}$ and $\Phi_{3}$ for domains $\mathrm{A}, \mathrm{B}$ and $\mathrm{C}$.

Following [12], under the uniform ensemble the coefficients $\left\{\Omega_{j}\right\}$ in the vorticity expansion (7) can be considered to be random variables. In the limit $N \rightarrow \infty$ the CLT can be applied to obtain the distribution of the $\left\{\Omega_{j}\right\}$. The result, which follows due to the choice of scalings made in section 2.1 , is that under the uniform ensemble

$$
\Omega_{j} \sim \mathcal{N}(0,1) \quad(j \geq 1)
$$

i.e. the $\left\{\Omega_{j}\right\}$ are independent identically distributed (i.i.d.) Gaussian random variables. (Independence follows from the orthogonality of the hydrodynamic modes.) Further, under the uniform ensemble, $\bar{H}$ is also a random variable, which by definition has the density of states function $W\left(\varepsilon, \Omega_{0}\right)$ as its pdf. By inspection of (12), in fact, $\bar{H}$ is a weighted sum of independent non-central chi-squared random variables. Weighted sums of this type have been studied in the statistics literature [5] and a brief guide to how they can be accurately evaluated in practice is given in Appendix B.

Using notation introduced in Appendix B for the result of the relevant random sum, the density of states is given by

$$
W\left(\varepsilon, \Omega_{0}\right)=F^{\left(\boldsymbol{\beta}, \Omega_{0}^{2} \boldsymbol{\mu}\right)}\left(\varepsilon-\varepsilon_{0}\left(\Omega_{0}\right)\right)
$$

where the function described by the notation $F^{(\boldsymbol{w}, \boldsymbol{\lambda})}(\cdot)$ is defined by $(62)$. Here $\boldsymbol{\beta}=-\left(\beta_{1}, \beta_{2}, \ldots\right)^{T}$ is (minus) the vector of hydrodynamic eigenvalues $\left\{\beta_{j}\right\}$, and $\boldsymbol{\mu}=\left(\mu_{1}^{2}, \mu_{2}^{2}, \ldots\right)^{T}$. Most of our results below are built on (18).

A natural idea, for the case of strongly non-neutral flows with $\Omega_{0}=$ $[\omega] / \Gamma_{0} N^{1 / 2} \gg 1$ is to simplify (18) by neglecting the quadratic terms (i.e. 
terms involving $\left.\Omega_{j}^{2}\right)$ in (12). Under this approximation $W\left(\varepsilon, \Omega_{0}\right)$ becomes a sum of Gaussian random variables and will consequently have a normal distribution. In fact, the most uniformly accurate Gaussian approximation of $W\left(\varepsilon, \Omega_{0}\right)$ is most easily obtained by replacing each non-central chi-squared random variable in (12) by a Gaussian random variable with zero mean and identical variance. Standard results for sums of Gaussian random variables then lead to

$$
W\left(\varepsilon, \Omega_{0}\right) \approx \frac{1}{\sqrt{2 \pi} \Lambda} \exp \left(-\frac{\left(\varepsilon-\varepsilon_{0}\left(\Omega_{0}\right)\right)^{2}}{2 \Lambda^{2}}\right), \quad \Lambda=\left(\sum_{j=1}^{\infty} \frac{1+2 \mu_{j}^{2} \Omega_{0}^{2}}{2 \beta_{j}^{2}}\right)^{1 / 2}
$$

The constant $\Lambda$, which gives the standard deviation of $W\left(\varepsilon, \Omega_{0}\right)$ depends only on the domain constants and $\Omega_{0}$. Note that $\Lambda \approx \Omega_{0}\left(\sum_{j} G_{0 j}^{2}\right)^{1 / 2}$ to leading order in $\Omega_{0}$. The advantage of the approximation (19) is that the formula for the energy spectrum etc. below can be evaluated exactly. The usefulness of the Gaussian approximation will be considered shortly.

In the upper panels of Fig. 1 the density of states $W\left(\varepsilon, \Omega_{0}\right)$, calculated from (18), is plotted (grey curves) for the Neumann oval domain illustrated in Fig. 2 (domain A). Details of the conformal map used to generate the Neumann oval domain are given in Refs. [13] and [12] (it is 'domain B' in the latter reference). The spectral methods described in $[13,12]$ are used to obtain the coefficients used to evaluate (18) numerically. The three panels show $\Omega_{0}=0$ (neutral case) $\Omega_{0}=5$ and $\Omega_{0}=10$. To verify the calculation in each case, also plotted are histogram approximations (solid points) to pdfs of $\bar{H}$ under the uniform ensemble, for $N=100$ vortices with circulations \pm 1 , with the number of positive vortices $N_{+}=50,75$ and 100 vortices respectively. The histograms are based on $10^{6}$ random samples of $\bar{H}$, with direct sampling used (as opposed to the Markov chain Monte-Carlo sampling method used in EA15). The lower panels of Fig. 1 show the inverse temperature $\beta=W^{\prime} / W$, where the prime denotes differentiation with respect to $\varepsilon$. In the neutral case, $\beta$ approaches a critical value $\beta_{c}=\beta_{1} \approx-42.61$, where $\beta_{1}$ is the leading eigenvalue of the hydrodynamic eigenvalue problem (38). The critical value $\beta_{c}$ is the inverse temperature at which Onsager-Kraichnan condensation occurs, i.e. a mean circulation emerges. At high energies, the mean circulation can be obtained by solving the sinh-Poisson (Boltzmann-Poisson) equation [15], which is derived using mean-field theory. The high $\varepsilon$ limit of the current theory, and the low energy limit of the mean-field theory can be reconciled by asymptotic matching, following [12]. In Fig. 1 it can be seen that in the non-neutral case the convergence $\beta \rightarrow \beta_{c}$ is much slower, which can be explained because the emergence of the condensate from the existing mean flow, which is present due to the mean vorticity field is rather gradual.

A shortcoming of the Gaussian approximation is apparent in the caloric curves plotted in Fig. 1. Under the Gaussian approximation the inverse temperature is linear

$$
\beta(\varepsilon) \approx-\frac{\varepsilon-\varepsilon_{0}\left(\Omega_{0}\right)}{\Lambda^{2}}
$$


which is evidently a poor approximation to the exact curves (plotted). In particular, the Gaussian approximation does not capture $\lim _{\varepsilon \rightarrow \infty} \beta=\beta_{c}$, which means that the Gaussian approximation cannot describe any aspect of condensation. For this reason it is not considered further below.

\subsection{Microcanonical statistics and the energy spectrum}

Useful statistical mechanical predictions, which under the ergodic hypothesis will correspond to the long-time means of the dynamics, are obtained from the microcanonical ensemble. In domains without additional symmetries, the microcanonical ensemble consists of all states in phase-space lying on the hypersurface of constant energy $\bar{H}=\varepsilon$.

For example, the microcanonical pdf of the projection $\Omega_{j}$ of the vorticity field onto the hydrodynamic mode $\Phi_{j}(\boldsymbol{x})$ can be obtained using Bayes' theorem. Using the subscript $\varepsilon$ to denote microcanonical pdfs, for $\Omega_{j}$ we have

$$
\begin{aligned}
p_{\varepsilon}\left(\omega_{j}\right) & =\frac{W^{(j)}\left(\varepsilon-\bar{e}_{j}\left(\omega_{j}\right), \Omega_{0}\right)}{W\left(\varepsilon, \Omega_{0}\right)} \frac{\exp \left(-\omega_{j}^{2} / 2\right)}{\sqrt{2 \pi}}, \\
\text { where } \bar{e}_{j}\left(\omega_{j}\right) & =\frac{\left(\omega_{j}+\Omega_{0} \mu_{j}\right)^{2}-1-\Omega_{0}^{2} \mu_{j}^{2}}{-2 \beta_{j}}
\end{aligned}
$$

Here $W^{(j)}\left(\varepsilon, \Omega_{0}\right)=F^{\left(\boldsymbol{\beta}, \Omega_{0}^{2} \boldsymbol{\mu}\right)(j)}\left(\varepsilon-\varepsilon_{0}\left(\Omega_{0}\right)\right)$ denotes the density of states function (18) modified so that it is calculated with the $j$ th mode omitted (for a description of the notation see Appendix B). The use of Bayes' theorem is apparent if one considers $p_{\varepsilon}\left(\omega_{j}\right)$ as a conditional pdf in the uniform ensemble, i.e. the pdf of $\Omega_{j}$ conditioned on $\bar{H}=\varepsilon$. Then, the various factors on the righthand side of (21) can be identified as the pdf of $\bar{H}$ conditioned on $\Omega_{j}=\omega_{j}$ $\left(W^{(j)}\right.$ term), the pdf of $\bar{H}$ in the uniform ensemble ( $W$ term) and the pdf of $\Omega_{j}$ in the uniform ensemble (Gaussian term).

Similarly, the corresponding expression for the pdf of the energy $E_{j}$ in the $j$ th mode is

$$
p_{\varepsilon}\left(e_{j}\right)=\frac{W^{(j)}\left(\varepsilon-\left(e_{j}-E_{0 j}\right), \Omega_{0}\right)}{W\left(\varepsilon, \Omega_{0}\right)} \bar{p}_{j}\left(e_{j}\right),
$$

where $\bar{p}_{j}(\cdot)$ is the pdf of the non-central chi-squared random variable defined by equation (57), and the constant $E_{0 j}$ is given by (15). The equilibrium energy spectrum can be obtained from $p_{\varepsilon}\left(e_{j}\right)$, by definition, as the expected energy in each mode under the microcanonical ensemble

$$
\left\langle E_{j}\right\rangle=\int_{0}^{\infty} e_{j} p_{\varepsilon}\left(e_{j}\right) \mathrm{d} e_{j} .
$$

The integral (23) can be expressed purely in terms of the density of states function, using the convolution result (59), together with the fact that

$$
\left(W^{(j)} * p_{j}\right)\left(\varepsilon, \Omega_{0}\right)=W\left(\varepsilon, \Omega_{0}\right)
$$



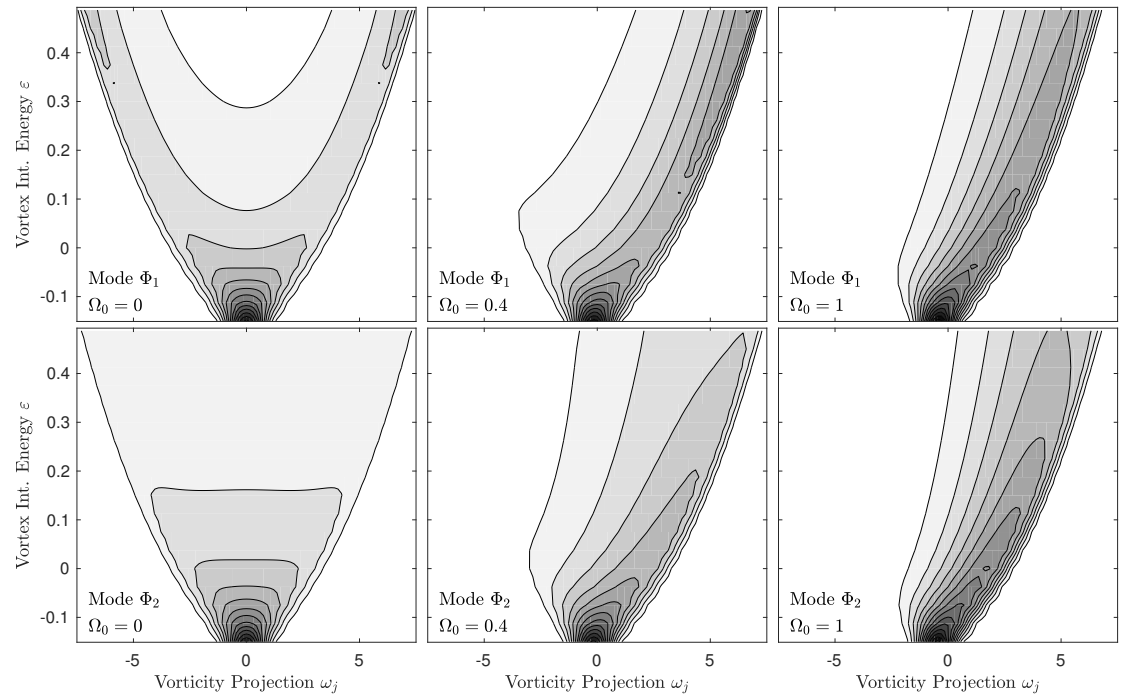

Fig. 3 For domain B, the microcanonical pdf $p_{\varepsilon}\left(\omega_{j}\right)$ of $\Omega_{j}$ at interaction energy $\varepsilon$, contoured as a function of $\left(\omega_{j}, \varepsilon\right)$, as predicted by $(21)$. Upper panels: $p_{\varepsilon}\left(\omega_{1}\right)$ for $\Omega_{0}=0,0.4,1$. Lower panels: $p_{\varepsilon}\left(\omega_{2}\right)$ for $\Omega_{0}=0,0.4,1$
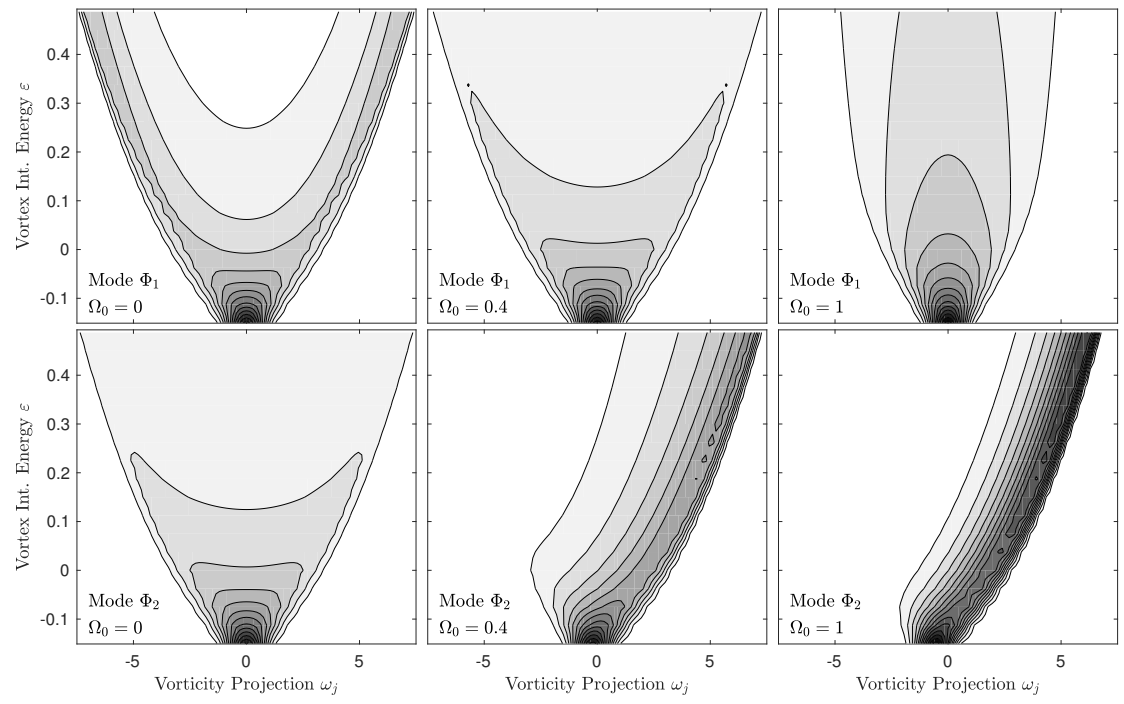

Fig. 4 As Figure 3 but for domain C.

These results lead directly to our main result for the equilibrium energy spectrum, generalising the result (2) for the neutral case, which is that at energy 

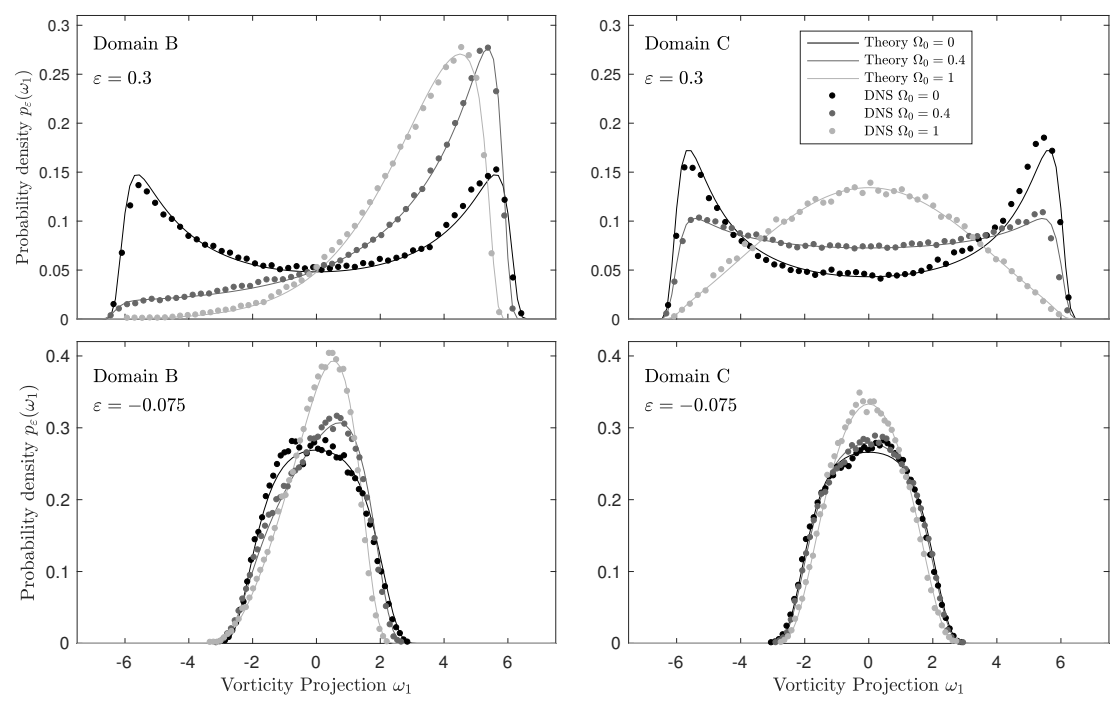

Fig. 5 Probability density $p_{\varepsilon}\left(\omega_{j}\right)$ of the vorticity projections $\Omega_{j}$ (i) predictions under the microcanonical ensemble from (21) (solid curves), (ii) histograms calculated from DNS results (points). Results from Domain B (left panels) and Domain C (right panels) and at $\varepsilon=0.3$ (upper panels) and at $\varepsilon=-0.075$ (lower panels) are shown. Results are for the neutral case $\left(\Omega_{0}=0, N_{+}=50, N_{-}=50\right.$, black curves), a weakly non-neutral case $\left(\Omega_{0}=0.4, N_{+}=52, N_{-}=48\right.$, dark grey curves), and a more strongly non-neutral case ( $\Omega_{0}=1, N_{+}=55, N_{-}=45$, light grey curves).

$\varepsilon$,

$$
\left\langle E_{j}\right\rangle=\frac{1}{2 W\left(\varepsilon, \Omega_{0}\right)} \int_{-\infty}^{\varepsilon} W\left(\bar{\varepsilon}, \Omega_{0}\right)\left(1-\Omega_{0}^{2} \mu_{j}^{2} \beta_{j}(\varepsilon-\bar{\varepsilon})\right) \mathrm{e}^{\beta_{j}(\varepsilon-\bar{\varepsilon})} \mathrm{d} \bar{\varepsilon} .
$$

Equation (24) shows that the energy spectrum is completely determined by the density of states function $W\left(\varepsilon, \Omega_{0}\right)$, and the energy in each mode can be obtained by a convolution of $W\left(\varepsilon, \Omega_{0}\right)$ with an elementary function. The significance of this is that the energy spectrum at all energies $\varepsilon$ can be obtained following a single calculation of $W\left(\varepsilon, \Omega_{0}\right)$. Recall also that $W\left(\varepsilon, \Omega_{0}\right)$ depends only on the shape of the domain $\mathcal{D}$ and not on any detail of the vortex circulations $\left(\Gamma_{1}, \ldots, \Gamma_{N}\right)$ apart from $\Omega_{0}$. Finally, it is interesting to note that $\left\langle E_{j}\right\rangle$ is determined by the structure of $W$ at energies below that of the energy $\varepsilon$ of interest only.

\subsection{Comparison with direct numerical simulations}

Direct numerical simulations (DNS) can be used to assess the accuracy of the predictions made in section 2.3. Details of the adaptive numerical scheme used 
to solve the equations of motion

$$
\Gamma_{i} \frac{\mathrm{d} x_{i}}{\mathrm{~d} t}=-\frac{\partial H}{\partial y_{i}}, \quad \Gamma_{i} \frac{\mathrm{d} y_{i}}{\mathrm{~d} t}=\frac{\partial H}{\partial x_{i}}, \quad i=1, \ldots, N
$$

are given in [1]. Here, very long integrations with $N=100$ vortices are performed (length $10^{5} \Gamma_{0}^{-1}$ ), and vortex positions recorded at interval $\Gamma_{0}^{-1}$ in order to obtain converged statistics. Non-neutrality is introduced by varying the respective numbers of positive and negative vortices, with $\left(N_{+}, N_{-}\right)=(50,50)$, $(52,48)$ and $(55,45)$ respectively, corresponding to $\Omega_{0}=0,0.4$ and 1 as in Figs. 3 and 4 . Integrations are performed for both domains $\mathrm{B}$ and $\mathrm{C}$, and for high $(\varepsilon=0.3)$ and low $(\varepsilon=-0.075)$ energies respectively, giving 12 integrations in total.

The main statistic to be tested is the microcanonical pdf $p_{\varepsilon}\left(\omega_{1}\right)$ of the vorticity projection

$$
\Omega_{1}(t)=\frac{\left[\omega \Phi_{1}\right]}{\omega_{0}}=\frac{1}{\Gamma_{0} N^{1 / 2}} \sum_{i=1}^{N} \Gamma_{i} \Phi_{1}\left(\boldsymbol{x}_{i}(t)\right) .
$$

Recall that, were the vortices distributed randomly in $\mathcal{D}$ (under the uniform ensemble), the pdf of $\Omega_{1}$ would be normally distributed $\sim \mathcal{N}(0,1)$. In practice, the projection $\Omega_{1}$ is calculated from the DNS results by cubic interpolation of the (gridded) spectral solution for $\Phi_{1}$ onto the vortex positions, with the interpolation performed in the conformal (circle) domain.

Fig. 5 shows histograms (points) generated from the 12 DNS integrations, versus the corresponding predictions (curves) obtained from (21). Excellent agreement is obtained for all 12 simulations, demonstrating that the theory correctly describes the influence of non-neutrality across a range of energies. The two domains also exhibit different behaviours when non-neutrality is introduced, exactly as predicted in Figs. 3-4.

\subsection{Saturation energy spectrum}

One of the main results of EA15 was to show that, in the neutral case, for large $\varepsilon$ the energy spectrum becomes saturated for all modes with $j \geq 2$. Following this saturation, as $\varepsilon$ is increased further, all of the additional energy must enter the $j=1$ (Onsager-Kraichnan) condensate mode. The main results can be extended to the non-neutral case by noting that as $\varepsilon \rightarrow \infty, \beta(\varepsilon) \rightarrow \beta_{1}$ and $W(\varepsilon) \sim \exp \left(\beta_{1} \varepsilon\right)$. Inserting this form into (23), gives, for $j \geq 2$

$$
\left\langle E_{j}\right\rangle \rightarrow \frac{\beta_{1}-\beta_{j}\left(1+\Omega_{0}^{2} \mu_{j}^{2}\right)}{2\left(\beta_{1}-\beta_{j}\right)^{2}} \quad(j \geq 2) .
$$

The energy in the condensate mode $(j=1)$ can then be obtained from the ensemble average of (12),

$$
\varepsilon=\varepsilon_{0}\left(\Omega_{0}\right)+\sum_{j=1}^{\infty}\left\langle E_{j}\right\rangle-E_{0 j},
$$


from which

$$
\left\langle E_{1}\right\rangle \sim \varepsilon-\varepsilon_{*}, \quad \text { where } \varepsilon_{*}=\varepsilon_{0}\left(\Omega_{0}\right)+\sum_{j=2}^{\infty} \frac{\beta_{1}\left(1+\Omega_{0}^{2} \mu_{j}^{2}\right)}{2 \beta_{j}\left(\beta_{1}-\beta_{j}\right)} .
$$

The main point to note from $(26-28)$ is that for $\left|\Omega_{0}\right| \gtrsim 1$ the non-neutral energy spectrum saturates at a considerably higher energy compared to the neutral case.

\section{Domains with translational and rotational symmetries}

\subsection{General considerations}

A domain $\mathcal{D}$ with an additional symmetry will have a conserved integral of the form

$$
I=\frac{1}{A} \int_{\mathcal{D}} F(\boldsymbol{x}) \omega(\boldsymbol{x}) \mathrm{d} \boldsymbol{x}
$$

in addition to the integral of the vorticity itself. The microcanonical ensemble for point vortex motion in $\mathcal{D}$ then consists of those states in phase-space on the hypersurface defined by $\bar{H}=\varepsilon$ and $I=$ constant. An explicit example is when $\mathcal{D}$ is chosen to be the unit circle, as will be explored in detail below. For the circle, $F(\boldsymbol{x})=|\boldsymbol{x}|^{2} / 2$, and $I$ is the familiar angular impulse, which is also conserved when $\mathcal{D}$ is annular. The general methodology applies equally to other domains, for example when $\mathcal{D}$ is a $\left(\boldsymbol{e}_{i^{-}}\right)$periodic channel bounded by sidewalls, in which case $F(\boldsymbol{x})=\left(\boldsymbol{e}_{3} \times \boldsymbol{x}\right) \cdot \boldsymbol{e}_{i}$ and $I$ is the linear impulse in the direction $\boldsymbol{e}_{i}(i=1,2)$. Other examples are possible if the class of domains is extended to include general two-dimensional surfaces, following [8].

The key to dealing with the additional conserved quantity is to introduce a generalised hydrodynamic basis as defined in appendix A. The expansion of the normalised vorticity field in this basis is

$$
\frac{\omega(\boldsymbol{x}, t)}{\omega_{0}}=\Omega_{0}+\Omega_{1} \Phi_{1}(\boldsymbol{x})+\sum_{j=2}^{\infty} \Omega_{j}(t) \Phi_{j}(\boldsymbol{x}) .
$$

The expansion is similar to (7), except that the first two coefficients $\Omega_{0}$ and $\Omega_{1}$ are now time-independent, reflecting the fact that there are now two conserved quantities. (Explicitly $\Omega_{0}=[\omega] / \omega_{0}, \Phi_{1}=(F-[F]) /\left[(F-[F])^{2}\right]^{1 / 2}$ and $\Omega_{1}=$ $\left.\left[\omega \Phi_{1}\right] / \omega_{0}.\right)$ The Dirichlet Green's function can then also be expanded in the generalised basis according to (53). Following the method of section 2.1, using the properties of the generalised basis established in appendix A, it follows that (10) becomes

$$
\bar{H}=\varepsilon_{0}\left(\Omega_{0}, \Omega_{1}\right)+\sum_{j=1}^{\infty} \frac{\left(\Omega_{j}+\mu_{j} \Omega_{0}+\nu_{j} \Omega_{1}\right)^{2}-\left(\mu_{j} \Omega_{0}+\nu_{j} \Omega_{1}\right)^{2}-1}{-2 \beta_{j}},
$$


with sequences of constants $\left\{\beta_{j}\right\},\left\{\mu_{j}\right\}$ and $\left\{\nu_{j}\right\}$ as defined in appendix A. Here

$$
\varepsilon_{0}\left(\Omega_{0}, \Omega_{1}\right)=-\frac{1}{2}\left(r_{0}+G_{00}\left(\Omega_{0}^{2}-1\right)+2 G_{01} \Omega_{0} \Omega_{1}+G_{11}\left(\Omega_{1}^{2}-1\right)\right) .
$$

In the uniform ensemble, equation (31) can be treated exactly as (12), as a sum of non-central chi-squared random variables. It follows that the formula for the density of states is

$$
W\left(\varepsilon, \Omega_{0}, \Omega_{1}\right)=F^{(\boldsymbol{\beta}, \boldsymbol{v})}\left(\varepsilon-\varepsilon_{0}\right)
$$

where, as in section 2 , the function described by the notation $F^{(\boldsymbol{w}, \boldsymbol{\lambda})}(\cdot)$ is defined by (62). In this case $\boldsymbol{\beta}=-\left(\beta_{2}, \beta_{3}, \ldots\right)^{T}$ is (minus) the vector of generalised hydrodynamic eigenvalues $\left\{\beta_{j}\right\}$, and $\boldsymbol{v}\left(\Omega_{0}, \Omega_{1}\right)=\left(\left(\Omega_{0} \mu_{2}+\Omega_{1} \nu_{2}\right)^{2},\left(\Omega_{0} \mu_{3}+\right.\right.$ $\left.\left.\Omega_{1} \nu_{3}\right)^{2}, \ldots\right)^{T}$. Using (32) further results corresponding to those in section 2 follow straightforwardly.

\subsection{Circular domains}

The circle is the simplest example of a domain with a rotational symmetry. The associated conserved quantity is the angular impulse (29) with $F(\boldsymbol{x})=\frac{1}{2}|\boldsymbol{x}|^{2}$. The circle of unit radius, which may be used here without loss of generality, has a well-known [3] Dirichlet Green's function

$$
G\left(\boldsymbol{x}, \boldsymbol{x}^{\prime}\right)=\frac{1}{2 \pi} \log \left|\boldsymbol{x}-\boldsymbol{x}^{\prime}\right|-\frac{1}{4 \pi} \log \left(1-2 \boldsymbol{x} \cdot \boldsymbol{x}^{\prime}+|\boldsymbol{x}|^{2}\left|\boldsymbol{x}^{\prime}\right|^{2}\right),
$$

from which all of the constants necessary to evaluate (32) can be calculated explicitly.

In terms of the usual polar coordinates $(r, \theta)$, the first two hydrodynamic eigenmodes are

$$
\Phi_{0}=1, \quad \Phi_{1}=2 \sqrt{3}\left(r^{2}-\frac{1}{2}\right) .
$$

The remaining eigenmodes $\left\{\Phi_{j}, j \geq 2\right\}$ consist of the union of the set of eigenmodes $S^{\theta}=\left\{\phi_{m n \pm}, m, n \geq 1\right\}$ with azimuthal dependence,

$$
\phi_{m n \pm}(r, \theta)=\frac{\sqrt{2} \mathrm{~J}_{m}\left(j_{m n} r\right)}{\mathrm{J}_{m+1}\left(j_{m n}\right)}\left\{\begin{array}{l}
\cos (m \theta) \\
\sin (m \theta)
\end{array}\right\}, \quad \beta_{m n}=-\pi j_{m n}^{2},
$$

with the $\theta$-independent set $S^{0}=\left\{\phi_{0 n}, n \geq 1\right\}$

$$
\phi_{0 n}(r)=\frac{j_{3 n} \mathrm{~J}_{0}\left(j_{3 n} r\right)-2 \mathrm{~J}_{1}\left(j_{3 n}\right)}{j_{3 n} \mathrm{~J}_{2}\left(j_{3 n}\right)}, \quad \beta_{0 n}=-\pi j_{3 n}^{2} .
$$

Here $\mathrm{J}_{m}(\cdot)$ is the Bessel function of the first kind of index $m$, and $j_{m n}$ denotes its zeroes $(n \geq 1)$. The axisymmetric eigenmodes in $S^{0}$ are most easily obtained by solving (51). The union of these sets of eigenmodes $\left\{\Phi_{j}, j \geq 2\right\}=S^{0} \cup S^{\theta}$ 


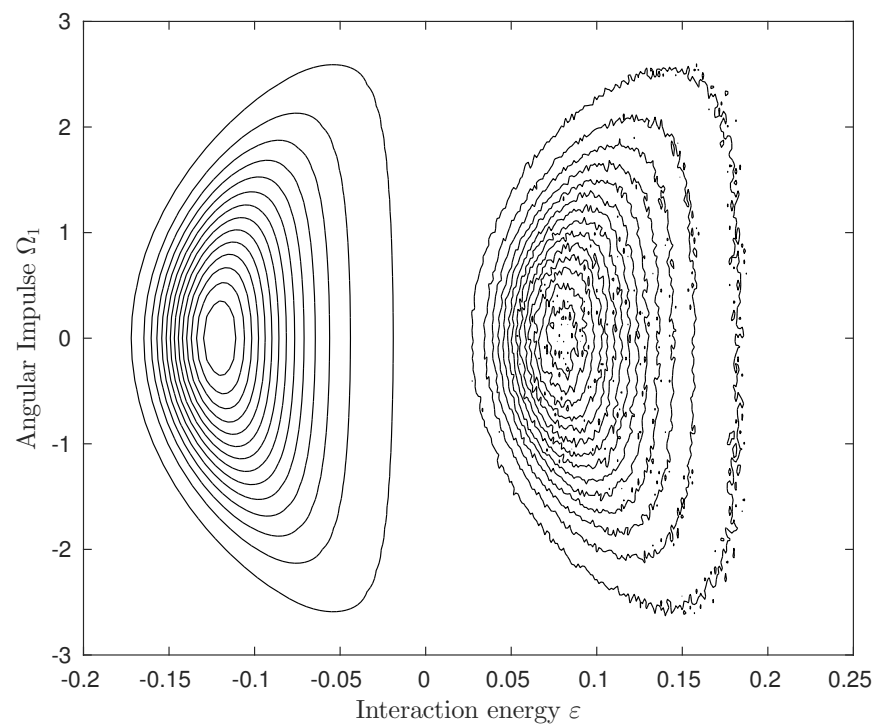

Fig. 6 The joint pdf $p_{0}\left(\varepsilon, \omega_{1}\right)$ of $\left(\bar{H}, \Omega_{1}\right)$, for a neutral vortex gas in the unit circle, under the uniform distribution. Left: Theoretical calculation from (32) (see text). Right: (shifted $\varepsilon \rightarrow \varepsilon+0.2)$ Histogram generated from $10^{6}$ random distributions of $N=100$ vortices.

are then ordered according to their eigenvalues $\left\{\beta_{j}, j \geq 2\right\}$. It is straightforward to verify the orthogonality condition $\left[\Phi_{j} \Phi_{k}\right]=\delta_{j k}$ for all $j, k \geq 0$ by direct calculation using Bessel function identities.

The remaining constants in (31) can also be evaluated straightforwardly for the circle. They are

$$
\mu_{j}=G_{0 j} \beta_{j}=0, \quad \nu_{j}=G_{1 j} \beta_{j}=\left\{\begin{array}{cl}
\frac{1}{\sqrt{3}}, & \Phi_{j} \in S^{0} \\
0, & \Phi_{j} \in S^{\theta}
\end{array} \quad(j \geq 2)\right.
$$

and

$$
G_{00}=-\frac{1}{8 \pi}, \quad G_{01}=\frac{1}{8 \sqrt{3} \pi}, \quad G_{11}=-\frac{1}{16 \pi}, \quad r_{0}=\frac{1}{2 \pi} .
$$

The results (35-37) allow the all of the statistical quantities introduced in section 2 to be calculated explicitly for the circle, with the only approximations being in the (necessary) truncation of summations, as described in appendix B. To test the accuracy of (32) Fig. 6 shows the following calculation. On the left, the joint pdf $p_{0}\left(\varepsilon, \omega_{1}\right)$ of $\left(\bar{H}, \Omega_{1}\right)$, for a neutral vortex gas under the uniform distribution, is contoured. The theoretical prediction is obtained using $p_{0}\left(\varepsilon, \omega_{1}\right)=W\left(\varepsilon ; 0, \omega_{1}\right) p_{0}\left(\omega_{1}\right)$ where $p_{0}\left(\omega_{1}\right) \sim \mathcal{N}(0,1)$, and with $W$ calculated from (32). A two-dimensional histogram of $\left(\bar{H}, \Omega_{1}\right)$, generated from $10^{6}$ random distributions of $N=100$ vortices (for plotting purposes shifted by 


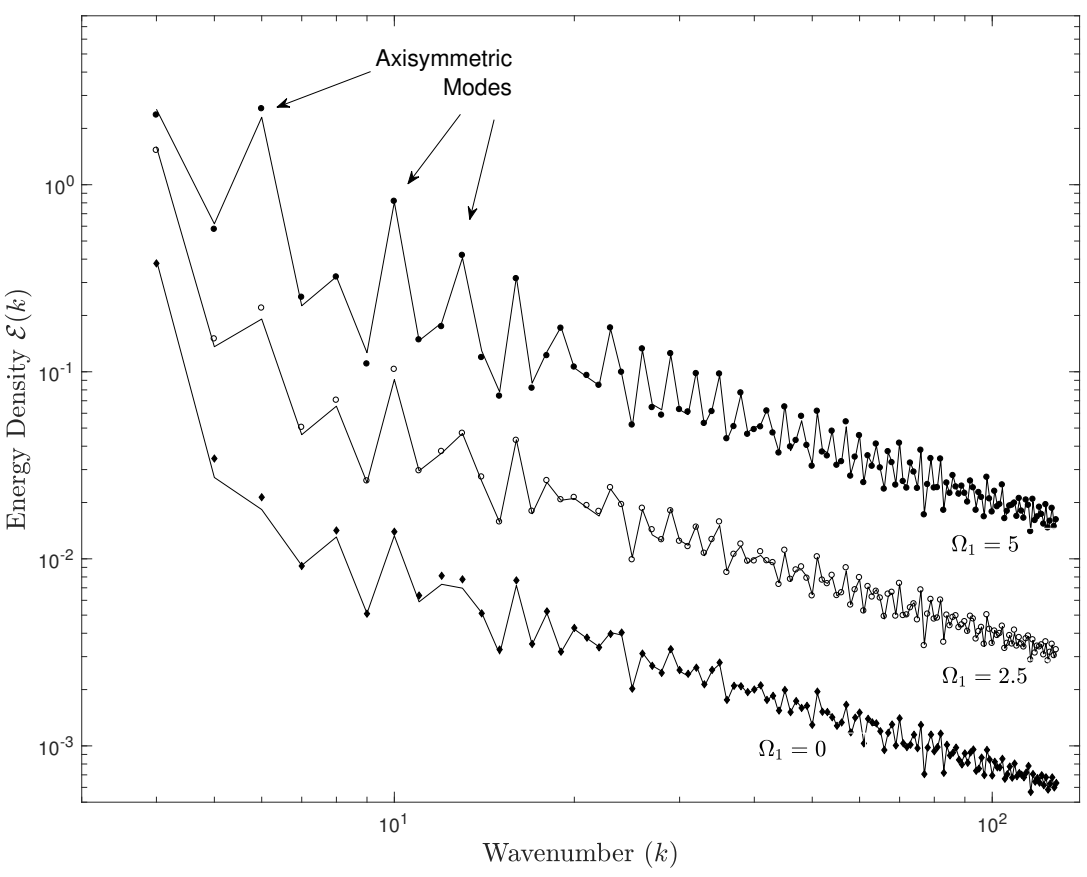

Fig. 7 Energy spectrum $\mathcal{E}(k)$ in the circle. Points show time-averages from three long $\left(100000 \Gamma_{0}^{-1}\right)$ DNS of $(25)$ with $N=100$ vortices, $\varepsilon=0.3$ and $\Omega_{1}=0,2.5$ and 5 respectively. Solid lines show the corresponding theoretical predictions from (24). For clarity, the results for $\Omega_{1}=2.5$ and 5 have been shifted upwards in the plot, by taking $\mathcal{E}(k) \rightarrow 5 \mathcal{E}(k)$ and $\mathcal{E}(k) \rightarrow 25 \mathcal{E}(k)$ respectively.

$\varepsilon \rightarrow \varepsilon+0.2$ ), is contoured on the right. Note that a similar histogram (without a corresponding theoretical description) has been calculated by [3]. Excellent agreement between the theoretical and sampled histograms is evident.

The resulting statistical predictions can be compared with dynamical integrations of (25). Three calculations of length $10^{5} \Gamma_{0}^{-1}$ have been performed in the circular geometry with $N=100$ vortices ( 50 positive, 50 negative, so that $\Omega_{0}=0$ ), each with $\varepsilon=0.3$, and with $\Omega_{1}=0,2.5$ and 5 respectively (note that angular impulse $I=\Omega_{1} \sqrt{N / 48}$ ).

In Fig. 7 predicted versus calculated energy spectra are compared for all three calculations. Following standard practice, the energy density $\mathcal{E}(k)$ is calculated for each integer wavenumber $k$ by summing the energies $E_{j}$ in all modes satisfying $k-\frac{1}{2}<k_{j} \leq k+\frac{1}{2}$, where $k_{j}=\left(-\beta_{j} / A\right)^{1 / 2}$. The energies $E_{j}(t)$ are themselves calculated directly from the vortex positions, using the definitions of $\Omega_{j}$, and $\Phi_{j}$ taken from (35-36). The theoretical predictions for $\left\langle E_{j}\right\rangle$ follow from adapting equation (24), using the substitution $\mu_{j} \Omega_{0} \rightarrow \nu_{j} \Omega_{1}$, and by using the relevant density of states (32). Good agreement is evident between theory and DNS. In particular, the theory captures the emergence of 
the spectral signature of the axisymmetric modes (i.e. those in $\mathcal{S}^{0}$, of which the first three are indicated) as $\Omega_{1}$ increases. It is notable that the increase in the energy of the axisymmetric modes is largely at the expense of the gravest modes in the system.

At larger values of $\varepsilon$ the energy of the flow becomes concentrated in an emergent condensate associated with these gravest modes, as can also be established using mean-field theory, following [15]. The structure and selection of the condensate in the circle has been studied in section 5 of [6], where a detailed description is given.

\section{Conclusions}

In this work, the statistical theory of [12] has been extended to cover the cases of both non-neutral vortex distributions and domains with additional symmetry-induced conserved quantities. In particular, we have shown that:

1. The density of states function for an arbitrary distribution of vortices, in an arbitrary domain $\mathcal{D} \subset \mathbb{R}^{2}$, can be calculated using (18).

2. A range of statistical quantities, such as the pdf $p_{\varepsilon}\left(\omega_{j}\right)$ of the vorticity projections $\Omega_{j}$ can be evaluated under the microcanonical ensemble. Under the ergodic hypothesis these pdfs are realised by the dynamics. Long numerical integrations with $N=100$ show excellent agreement with the predictions for both neutral and non-neutral vortex distributions.

3. A simple expression (24) for the microcanonical energy spectrum is obtained. Numerical integrations in the circle, again with $N=100$, show that the theory captures the variations in the energy spectrum as the vortex interaction energy $\varepsilon$, and angular momentum $I$ are varied.

4. The theory is naturally extended to domains with conserved quantities. Numerical integrations with $N=100$ in the unit circle have been used to verify the results.

The results above will be of particular use to researchers interested in twodimensional quantum turbulence $[22,2]$ for two reasons. First, a number of experiments with Bose-Einstein condensates have been undertaken in an annular trapping potential [17], which requires consideration of the angular momentum constraint. Second, quantised vortices modelled by the Gross-Pitaevskii equation undergo like-signed annihilation, which in a double-periodic domain preserves neutrality. In a bounded domain, however, annihilation can also occur with the 'image' vortex at the boundary, meaning that non-neutral configurations can emerge spontaneously from the dynamics [21]. The results here allow the equilibrium statistics to be determined in each of the above situations. 
A The hydrodynamic basis and its generalisation

A.1 The hydrodynamic basis and the expansion of the Dirichlet Green's function

Ideal hydrodynamics in a simply-connected, bounded domain $\mathcal{D}$ conserves the integral of the vorticity. Assuming that there are no other conserved quantities that are linear in the vorticity, a natural basis in which to expand the residual vorticity field $\omega-[\omega]$ will consist of eigenmodes $\left\{\Phi_{j}\right\}$ which carry no vorticity, i.e. which satisfy $\left[\Phi_{j}\right]=0$. It is known that solutions of the eigenvalue problem

$$
\nabla^{2} \Phi=\frac{\beta}{A} \Phi \text { in } \mathcal{D}
$$

subject to boundary conditions

$$
\Phi=\text { cons. on } \partial \mathcal{D}, \quad \oint_{\partial \mathcal{D}} \nabla \Phi \cdot \boldsymbol{n} \mathrm{d} s=0
$$

satisfy this condition. The boundary conditions (39) are known as hydrodynamic boundary conditions, and their role in $2 \mathrm{D}$ vorticity dynamics is long recognised $[16,14]$. Note if $\Phi_{j}$ is interpreted as a streamfunction, then the boundary integral in (39) can be interpreted as enforcing a zero circulation condition on $\partial \mathcal{D}$, which is consistent with the mode carrying no vorticity (c.f. Kelvin's circulation theorem). Each mode $\Phi_{j}$ has an associated non-positive eigenvalue $\beta_{j}$. Here, use of the domain area $A$ in (38) ensures that the $\left\{\beta_{j}\right\}$ depend only on the shape and not the size of $\mathcal{D}$. The modes are orthogonal and can be normalised (as here) so that $\left[\Phi_{j} \Phi_{k}\right]=\delta_{j k}$ where [.] denotes the domain average. Further $\Phi_{0}=1$, with eigenvalue $\beta_{0}=0$, and it follows that $\left[\Phi_{j}\right]=0$ for all $j \geq 1$. $[6]$

A different perspective on (38-39) is provided by the closely related eigenvalue problem

$$
\nabla^{2} \Psi=\frac{\beta}{A}(\Psi-[\Psi]), \quad \text { with } \Psi=0 \text { on } \partial \mathcal{D},
$$

which shares eigenvalues $\left\{\beta_{j}\right\}$ with (38-39) for $j \geq 1$, note that $\Phi_{j}$ can be identified with $\nabla^{2} \Psi_{j}$. A near-equivalent integral equation representation of (40) is

$$
\begin{aligned}
\mathcal{L} \Phi & =\beta^{-1} \Phi \\
\mathcal{L} f(\boldsymbol{x}) & =\frac{1}{A} \int_{\mathcal{D}} G_{H}\left(\boldsymbol{x}, \boldsymbol{x}^{\prime}\right) f\left(\boldsymbol{x}^{\prime}\right) \mathrm{d} \boldsymbol{x}^{\prime} \\
\text { where } \quad & \\
\text { and } \quad G_{H}\left(\boldsymbol{x}, \boldsymbol{x}^{\prime}\right) & =G\left(\boldsymbol{x}, \boldsymbol{x}^{\prime}\right)-G_{0}(\boldsymbol{x})-G_{0}\left(\boldsymbol{x}^{\prime}\right)+G_{00} .
\end{aligned}
$$

Here $G\left(\boldsymbol{x}, \boldsymbol{x}^{\prime}\right)$ is the Dirichlet Green's function and $G_{0}(\boldsymbol{x})=\left[G\left(\boldsymbol{x}, \boldsymbol{x}^{\prime}\right)\right]^{\prime}$ and $G_{00}=\left[\left[G\left(\boldsymbol{x}, \boldsymbol{x}^{\prime}\right)\right]\right]^{\prime}$ its domain averages over one and both variables respectively. The equivalence between (40) and (41) can be seen by substituting the relation

$$
\Psi(\boldsymbol{x})=\int_{\mathcal{D}} G\left(\boldsymbol{x}, \boldsymbol{x}^{\prime}\right) \Phi\left(\boldsymbol{x}^{\prime}\right) \mathrm{d} \boldsymbol{x}^{\prime}
$$

into (40). Notice it follows from the symmetry of $G$ that $G_{H}$ is symmetric, and therefore the operator $\mathcal{L}$ is self-adjoint and has real eigenvalues and orthogonal eigenmodes.

An expansion of the Dirichlet Green's function $G\left(\boldsymbol{x}, \boldsymbol{x}^{\prime}\right)$ in the complete set of modes $\left\{\Phi_{j}\right\}$ is used in section 2. In general, the expansion has the form

$$
G\left(\boldsymbol{x}, \boldsymbol{x}^{\prime}\right)=\sum_{j=0}^{\infty} \sum_{k=0}^{\infty} G_{j k} \Phi_{j}(\boldsymbol{x}) \Phi_{k}\left(\boldsymbol{x}^{\prime}\right)
$$


with the $\left\{G_{j k}\right\}$ given by (14). However, (42) can be simplified, because, since the $\left\{\Phi_{j}\right\}$ are eigenfunctions of $\mathcal{L}$ and $\Phi_{0}=1$ is in the kernel of $\mathcal{L}$,

$$
G_{H}\left(\boldsymbol{x}, \boldsymbol{x}^{\prime}\right)=\sum_{j=1}^{\infty} \frac{\Phi_{j}(\boldsymbol{x}) \Phi_{j}\left(\boldsymbol{x}^{\prime}\right)}{\beta_{j}} .
$$

Inserting (42) and (43) into the definition of $G_{H}$ in (41) it follows that $G_{j k}=\delta_{j k} / \beta_{j}$ for $j, k \geq 1$, and

$$
G\left(\boldsymbol{x}, \boldsymbol{x}^{\prime}\right)=G_{00}+\sum_{j=1}^{\infty} \frac{\Phi_{j}(\boldsymbol{x}) \Phi_{j}\left(\boldsymbol{x}^{\prime}\right)+\mu_{j} \Phi_{j}(\boldsymbol{x})+\mu_{j} \Phi_{j}\left(\boldsymbol{x}^{\prime}\right)}{\beta_{j}}
$$

with the $\left\{\beta_{j}\right\}$ and the $\left\{\mu_{j}\right\}$ the two infinite sequences of domain-shape dependent constants, given by (13).

The definition (15) of the energy spectrum $\left\{E_{j}\right\}$ is justified as follows. The definition of the fluid energy $E$ in the case of a non-singular streamfunction $\psi$ is

$$
E=\frac{1}{2} \int_{\mathcal{D}} \nabla \psi \cdot \nabla \psi \mathrm{d} \boldsymbol{x}
$$

Any streamfunction

$$
\psi(\boldsymbol{x})=\int_{\mathcal{D}} G\left(\boldsymbol{x}, \boldsymbol{x}^{\prime}\right) \omega\left(\boldsymbol{x}^{\prime}\right) \mathrm{d} \boldsymbol{x}^{\prime}+\text { constant, }
$$

can be expanded in the hydrodynamic eigenfunctions as $\psi=\omega_{0}^{2} \sum_{j=1}^{\infty} a_{j} \Phi_{j}$, omitting the $j=0$ term on account of the arbitrary constant and introducing a scaling factor $\omega_{0}$ for the vorticity, such as that used in section 2.1. Based on this expansion it is natural to define the scaled energy spectrum $\left\{E_{j}\right\}$, where $E=\omega_{0}^{2} \sum_{j=1}^{\infty} E_{j}$, to be

$$
E_{j}=\frac{a_{j}^{2}}{2} \int_{\mathcal{D}} \nabla \Phi_{j} \cdot \nabla \Phi_{j} \mathrm{~d} \boldsymbol{x}=-\frac{a_{j}^{2}}{2 \beta_{j}}
$$

using integration by parts with (38-39). Returning to the point vortex system, inserting the expansions (7) and (44) into (46) gives $a_{j}=\Omega_{j}+\mu_{j} \Omega_{0}$, recovering (15).

\section{A.2 The generalized hydrodynamic basis}

The hydrodynamic basis defined above is the appropriate basis when $\mathcal{D}$ has no continuous symmetries. If $\mathcal{D}$ has such a symmetry there will be an additional conserved quantity of the form (29). In this case, a generalised hydrodynamic basis $\left\{\Phi_{j}\right\}$ can be constructed by first defining $\Phi_{0}=1$ and $\Phi_{1}=(F-[F]) /\left[(F-[F])^{2}\right]^{1 / 2}$.

Next consider the eigenvalue problem

$$
\begin{aligned}
\mathcal{L} \Phi & =\beta^{-1} \Phi \\
\text { where } \quad \mathcal{L} f(\boldsymbol{x}) & =\frac{1}{A} \int_{\mathcal{D}} G_{H}\left(\boldsymbol{x}, \boldsymbol{x}^{\prime}\right) f\left(\boldsymbol{x}^{\prime}\right) \mathrm{d} \boldsymbol{x}^{\prime}
\end{aligned}
$$

where this time $G_{H}$ is obtained from the Dirichlet Green's function $G$ by

$$
\begin{aligned}
G_{H}\left(\boldsymbol{x}, \boldsymbol{x}^{\prime}\right)=G\left(\boldsymbol{x}, \boldsymbol{x}^{\prime}\right)-G_{0}(\boldsymbol{x})-G_{0}\left(\boldsymbol{x}^{\prime}\right)-\Phi_{1}(\boldsymbol{x}) G_{1}\left(\boldsymbol{x}^{\prime}\right)-G_{1}(\boldsymbol{x}) \Phi_{1}\left(\boldsymbol{x}^{\prime}\right) \\
+G_{01} \Phi_{1}\left(\boldsymbol{x}^{\prime}\right)+G_{01} \Phi_{1}(\boldsymbol{x})+G_{11} \Phi_{1}(\boldsymbol{x}) \Phi_{1}\left(\boldsymbol{x}^{\prime}\right)+G_{00}
\end{aligned}
$$

where $G_{j k}$ is defined as in $(14), G_{0}(\boldsymbol{x})=\left[G\left(\boldsymbol{x}, \boldsymbol{x}^{\prime}\right)\right]^{\prime}$ and $G_{1}(\boldsymbol{x})=\left[G\left(\boldsymbol{x}, \boldsymbol{x}^{\prime}\right) \Phi_{1}\left(\boldsymbol{x}^{\prime}\right)\right]^{\prime}$.

The eigenvalue problem (48) has the following properties: 
- It is straightforward to verify that the orthogonal functions $\Phi_{0}$ and $\Phi_{1}$ are in the kernel of $\mathcal{L}$, i.e $\mathcal{L} \Phi_{0}=\mathcal{L} \Phi_{1}=0$ so that both $\Phi_{0}$ and $\Phi_{1}$ are eigenmodes of $\mathcal{L}$ with eigenvalue $\beta^{-1}=0$.

- The modified Green's function $G_{H}\left(\boldsymbol{x}, \boldsymbol{x}^{\prime}\right)$ is symmetric and as a consequence $\mathcal{L}$ is selfadjoint. It follows that the remaining eigenmodes $\left\{\Phi_{j}, j \geq 2\right\}$ are orthogonal both to each other and to $\left\{\Phi_{0}, \Phi_{1}\right\}$. Further, they can be normalised so that

$$
\left[\Phi_{j} \Phi_{k}\right]=\delta_{j k} \quad j, k \geq 0 .
$$

- It follows, using the orthogonality property above, that the eigenmodes $\left\{\Phi_{j}, j \geq 2\right\}$ are also solutions of

$$
\nabla^{2} \Psi=\frac{\beta}{A}\left(\Psi-[\Psi]-\left[\Psi \Phi_{1}\right] \Phi_{1}\right), \quad \Psi=0 \text { on } \partial D, \text { where } \Psi=\int_{\mathcal{D}} G\left(\boldsymbol{x}, \boldsymbol{x}^{\prime}\right) \Phi\left(\boldsymbol{x}^{\prime}\right) \mathrm{d} \boldsymbol{x}^{\prime} .
$$

The eigenvalue problem in the form (51) has been previously identified as being key to understanding ideal fluid equilibrium states in the unit circle [6].

- The modified Green's function $G_{H}\left(\boldsymbol{x}, \boldsymbol{x}^{\prime}\right)$ can be expanded as

$$
G_{H}\left(\boldsymbol{x}, \boldsymbol{x}^{\prime}\right)=\sum_{j=2}^{\infty} \frac{\Phi_{j}(\boldsymbol{x}) \Phi_{j}\left(\boldsymbol{x}^{\prime}\right)}{\beta_{j}} .
$$

It follows that the corresponding expansion of the Dirichlet Green's function is

$$
\begin{aligned}
G\left(\boldsymbol{x}, \boldsymbol{x}^{\prime}\right) & =G_{00}+G_{01} \Phi_{1}(\boldsymbol{x})+G_{01} \Phi_{1}\left(\boldsymbol{x}^{\prime}\right)+G_{11} \Phi_{1}(\boldsymbol{x}) \Phi_{1}\left(\boldsymbol{x}^{\prime}\right) \\
& +\sum_{j=2}^{\infty} \frac{\Phi_{j}(\boldsymbol{x}) \Phi_{j}\left(\boldsymbol{x}^{\prime}\right)+\left(\mu_{j}+\nu_{j} \Phi_{1}(\boldsymbol{x})\right) \Phi_{j}\left(\boldsymbol{x}^{\prime}\right)+\left(\mu_{j}+\nu_{j} \Phi_{1}\left(\boldsymbol{x}^{\prime}\right)\right) \Phi_{j}(\boldsymbol{x})}{\beta_{j}}
\end{aligned}
$$

where $\mu_{j}=G_{0 j} / G_{j j}$ and $\nu_{j}=G_{1 j} / G_{j j}(j \geq 2)$.

When the expansion (53) is inserted into the Hamiltonian (10), along with corresponding expansions for the distributions $\omega, R$ and $S$, the result is equation (31).

\section{B Weighted sums of non-central chi-squared random variables}

As in EA15, it is necessary to introduce some functional notation to specify our results. Whereas in EA15 the results were expressed in terms of functions defined by weighted sums of $\chi$-squared random variables, here we need sums of non-central $\chi$-squared random variables. Such sums have been studied in the statistics literature [5], where it is shown that the resulting pdfs can be written in terms of (rather complicated) series involving Laguerre polynomials. Here, we present the simpler task of deriving the pdf as an inverse Fourier transform, which we approximate using numerical quadrature for the results in the main text.

The non-central chi-squared random variable, usually denoted $\chi_{1}^{\prime 2}(\lambda)$, is by definition equivalent to the random variable $Y=(X+\sqrt{\lambda})^{2}$ when $X \sim \mathcal{N}(0,1)$ is a Gaussian random variable and $\lambda>0$ a positive parameter. Explicitly, $Y$ has pdf

$$
p(y, \lambda)=\frac{H(y) \exp (-(y+\lambda) / 2) \cosh (\sqrt{\lambda y})}{\sqrt{2 \pi y}},
$$

where $H(\cdot)$ denotes the Heaviside step function. Note that $\mathbb{E}(Y)=1+\lambda$.

In the main text, the density of states function $W\left(\varepsilon, \Omega_{0}\right)$ is found, up to a shift in the independent variable, to be equal to the pdf of the random variable

$$
S_{M}=\sum_{j=1}^{M} \frac{Y_{j}-1-\lambda_{j}}{2 w_{j}},
$$


in the limit $M \rightarrow \infty$. Here $\left\{Y_{j}\right\}$ are independent and distributed according to $Y_{j} \sim \chi_{1}^{\prime 2}\left(\lambda_{j}\right)$, i.e. with pdf (54), where the $\left\{\lambda_{j}\right\}$ are a sequence of non-negative constants. The $\left\{w_{j}\right\}$ are an infinite set of monotonically increasing positive constants, which are assumed to increase sufficiently rapidly so that (a) CLT results for weighted sums (e.g. Lyapunov CLT) do not hold, and (b) $S_{\infty}=\lim _{M \rightarrow \infty} S_{M}$ is well defined. It turns out that $w_{j} \sim j$ at large $j$, which captures the behaviour of the eigenvalues $\left\{\beta_{j}\right\}$ in (12), is sufficient for both (a) and (b).

Denoting the pdf of $S_{M}$ by $F_{M}^{(\boldsymbol{w}, \boldsymbol{\lambda})}(s)$, where $\boldsymbol{w}=\left(w_{1}, w_{2}, \ldots, w_{M}\right)^{T}$ and $\boldsymbol{\lambda}=\left(\lambda_{1}, \lambda_{2}, \ldots, \lambda_{M}\right)^{T}$, it is a standard result that it can be expressed as a convolution

$$
F_{M}^{(\boldsymbol{w}, \boldsymbol{\lambda})}(s)=\left(p_{1} * p_{2} * p_{3} * \ldots * p_{M}\right)(s),
$$

where the $\left\{p_{j}\right\}$ are, in terms of (54), given by

$$
p_{j}(y)=2 w_{j} p\left(2 w_{j} y+1+\lambda_{j}, \lambda_{j}\right) .
$$

It is helpful for the analysis in 2.3 to also introduce the pdf of $Y_{j} / 2 w_{j}$ which is given explicitly by

$$
\bar{p}_{j}(y)=2 w_{j} p\left(2 w_{j} y, \lambda_{j}\right) .
$$

which is also related to $p_{j}$ by $\bar{p}_{j}(y)=p_{j}\left(y-\left(1+\lambda_{j}\right) / 2 w_{j}\right)$. A useful property of $\bar{p}_{j}$, which is straightforward to verify by direct integration of the convolution integral, is the result

$$
y \bar{p}_{j}(y)=\left(f * \bar{p}_{j}\right)(y) \text {, where } f(y)=\frac{1}{2} H(y)\left(1+\lambda_{j} w_{j} y\right) \exp \left(-w_{j} y\right) .
$$

Using the convolution theorem, the Fourier transform of $F_{M}^{(\boldsymbol{w}, \boldsymbol{\lambda})}(s)$ is given by

$$
\hat{F}_{M}^{(\boldsymbol{w}, \boldsymbol{\lambda})}(k)=(2 \pi)^{(M-1) / 2} \prod_{j=1}^{M} \hat{p}\left(\frac{k}{2 w_{j}} ; \lambda_{j}\right) .
$$

where

$$
\hat{p}(k ; \lambda)=\frac{1}{\sqrt{2 \pi}}\left(1+4 k^{2}\right)^{-1 / 4} \exp \left(-\frac{2 \lambda k^{2}}{1+4 k^{2}}+\frac{1}{2} \mathrm{i}\left(2 k-\tan ^{-1}(2 k)+\frac{8 \lambda k^{3}}{1+4 k^{2}}\right)\right) .
$$

is the Fourier transform of $p(y)$. Taking the limit $M \rightarrow \infty$, and dropping the subscript $M$, after some working it follows that

$$
F^{(\boldsymbol{w}, \boldsymbol{\lambda})}(s)=\frac{1}{2 \pi} \int_{-\infty}^{\infty} A^{(\boldsymbol{w}, \boldsymbol{\lambda})}(k) \exp \left(\mathrm{i} k s+\mathrm{i} \phi^{(\boldsymbol{w}, \boldsymbol{\lambda})}(k)\right) \mathrm{d} k,
$$

where the amplitude and phase functions $A^{(\boldsymbol{w}, \boldsymbol{\lambda})}$ and $\phi^{(\boldsymbol{w}, \boldsymbol{\lambda})}$ are real-valued and defined by

$$
\begin{aligned}
& A^{(\boldsymbol{w}, \boldsymbol{\lambda})}(k)=\exp \left(-\frac{1}{2} \sum_{j=1}^{\infty} \frac{k^{2} \lambda_{j}}{k^{2}+w_{j}^{2}}\right) \prod_{j=1}^{\infty}\left(1+\frac{k^{2}}{w_{j}^{2}}\right)^{-1 / 4}, \\
& \phi^{(\boldsymbol{w}, \boldsymbol{\lambda})}(k)=\frac{1}{2} \sum_{j=1}^{\infty}\left(\frac{k}{w_{j}}-\tan ^{-1}\left(\frac{k}{w_{j}}\right)+\frac{\lambda_{j} k^{3}}{w_{j}\left(k^{2}+w_{j}^{2}\right)}\right) .
\end{aligned}
$$

The function notation $F^{(\boldsymbol{w}, \boldsymbol{\lambda})}(s)$ is used in the main text. A further notational device used in the text is to write $(\boldsymbol{w}, \boldsymbol{\lambda})\left(i_{1}, i_{2}, i_{3}\right)$ to denote the vectors $\boldsymbol{w}$ and $\boldsymbol{\lambda}$ with the terms at index $j=i_{1}, i_{2}, i_{3}$ omitted (e.g. $(\boldsymbol{w}, \boldsymbol{\lambda})(1)=\left(w_{2} w_{3} \ldots\right)^{T}$ and $\left(\lambda_{2} \lambda_{3} \ldots\right)^{T}$ etc. $)$.

There is no known analytical method for explicitly inverting the transform (62). While Ref. [5] has shown that a series in Laguerre polynomials can be found for finite sums, this method becomes impractical as the number of non-central chi-squared random variables increases. As a consequence we use a direct numerical approach. Numerical quadrature of (62) using the trapezium rule, with $\boldsymbol{w}$ and $\boldsymbol{\lambda}$ truncated at a few hundred terms converges fairly rapidly with element size, provided that $s \lesssim 2\left(\sum_{j} w_{j}^{-2}\right)^{1 / 2}$ (roughly four standard deviations of the resulting distribution). Convergence is improved by using Weyl's law to approximate higher eigenvalues, and by using the Gaussian approximation (see main text) to include all eigenvalues beyond the cut-off in the calculation. Nevertheless as $|s|$ increases past $2\left|\sigma_{\varepsilon}\right|$ convergence rapidly becomes computationally expensive and then impossible. 
Acknowledgements Tom Ashbee is thanked for helpful comments.

\section{References}

1. Ashbee, T.L., Esler, J.G., McDonald, N.R.: Generalized Hamiltonian point vortex dynamics on arbitrary domains using the method of fundamental solutions. J. Comput. Phys. 246, 289-303 (2013)

2. Billam, T.P., Reeves, M.T., Anderson, B.P., Bradley, A.S.: Onsager-Kraichnan condensation in decaying two-dimensional quantum turbulence. Phys. Rev. Lett. 112, 145,301 (2014)

3. Bühler, O.: Statistical mechanics of strong and weak point vortices in a cylinder. Phys. Fluids 14(7), 2139-2149 (2002)

4. Campbell, L.J., O'Neil, K.: Statistics of two-dimensional point vortices and high-energy vortex states. J. Stat. Phys. 65, 495-529 (1991)

5. Castano-Martinez, A., Lopez-Blazquez, F.: Distribution of a sum of weighted noncentral chi-square variables. Test 14, $397 ? 415$ (2005)

6. Chavanis, P.H., Sommeria, J.: Classification of self-organized vortices in two-dimensional turbulence: The case of a bounded domain. J. Fluid Mech. 314, 267-297 (1996)

7. Crowdy, D.G., Kropf, E.H., Green, C.C., Nasser, M.M.S.: The Schottky-Klein prime function: a theoretical and computational tool for applications. IMA J. Appl. Math. 81, 589-628 (2016)

8. Dritschel, D.G., Boatto, S.: The motion of point vortices on compact surfaces. Proc. R. Soc. A 471, 20140,890 (2015)

9. Dritschel, D.G., Lucia, M., Poje, A.C.: Equilibrium statistics and dynamics of point vortex flows on the sphere. Phys. Rev. E 91, 063,014 (2015)

10. Dritschel, D.G., Scott, R.K., Macaskill, C., Gottwald, G.A., Tran, C.V.: Unifying scaling theory for vortex dynamics in two-dimensional turbulence. Phys. Rev. Lett. 101 094,501 (2008)

11. Esler, J.G.: The equilibrium energy spectrum of point vortex motion with remarks on ensemble choice and ergodicity. Phys. Rev. Fluids 2, 014,703 (2017)

12. Esler, J.G., Ashbee, T.L.: Universal statistics of point vortex turbulence. J. Fluid Mech. 779, 275-308 (2015)

13. Esler, J.G., Ashbee, T.L., McDonald, N.R.: Statistical mechanics of a neutral pointvortex gas at low energy. Phys. Rev. E 88, 012,109 (2013)

14. Flucher, M., Gustafsson, B.: Vortex motion in two dimensional hydrodynamics. Springer, New York (1999)

15. Joyce, G., Montgomery, D.: Negative temperature states for a two-dimensional guiding center plasma. J. Plasma Phys. 10, 107-121 (1973)

16. Lin, C.C.: On the motion of vortices in two dimensions-i. existence of the kirchhoff-routh function. Proc. Natl. Acad. Sci. 27(12), pp. 570-575 (1941)

17. Neely, T.W., Bradley, A.S., Samson, E.C., Rooney, S.J., Wright, E.M., Law, K.J.H., Carretero-González, R., Kevrekidis, P.G., Davis, M.J., Anderson, B.P.: Characteristics of two-dimensional quantum turbulence in a compressible superfluid. Phys. Rev. Lett. 111, 235,301 (2013)

18. Onsager, L.: Statistical hydrodynamics. Il Nuovo Cimento 6, 279-287 (1949)

19. Pointin, Y.B., Lundgren, T.S.: Statistical mechanics of two-dimensional vortices in a bounded container. Phys. Fluids 19(10), 1459-1470 (1976)

20. Reeves, M.T., Billam, T.P., Anderson, B.P., Bradley, A.S.: Signatures of coherent vortex structures in a disordered two-dimensional quantum fluid. Phys. Rev. A 89, 053,631 (2014)

21. Salman, H., Maestrini, D.: Long-range ordering of topological excitations in a twodimensional superfluid far from equilibrium. Phys. Rev. A 94, 043,642 (2016)

22. Simula, T., Davis, M.J., Helmerson, K.: Emergence of order from turbulence in an isolated planar superfluid. Phys. Rev. Lett. 113, 165,302 (2014)

23. Taylor, J.B., Borchardt, M., Helander, P.: Interacting vortices and spin-up in twodimensional turbulence. Phys. Rev. Lett. 102, 124,505 (2009)

24. Trefethen, L.N.: Spectral Methods in MATLAB. SIAM, Philadelphia (2000) 